\title{
Enhancement of the Critical Temperature Induced by the Quantum Size Effect in Superconducting Nanofilms
}

\author{
P. WÓJCIK* \\ AGH University of Science and Technology, Faculty of Physics and Applied Computer Science, \\ al. A. Mickiewicza 30, 30-059 Krakow, Poland
}

\begin{abstract}
The interplay between the quantum size effect and superconductivity in the metallic Al nanofilms has been studied with the use of the self-consistent numerical solutions of the Bogoliubov-de Gennes equations. We have shown that the critical temperature of the metallic nanofilm oscillates as a function of the nanofilm thickness. This phenomenon results from the quasi-particle energy quantization induced by the confinement of electrons in the direction perpendicular to the film. For the ultrathin nanofilms with thickness 1-2 $\mathrm{nm}$ we have found that the critical temperature increases up to value several times higher as compared to the one measured in the bulk.
\end{abstract}

DOI: 10.12693/APhysPolA.126.A-130

PACS: 74.78.-w, 73.61.-r, 74.78.Na

\section{Introduction}

Recent development in nanotechnology allows for the fabrication of structures with size of several nanometers such as nanowires [1-3] and nanofilms [4-6]. In these systems, the motion of electrons is confined to the nanometer scale leading to the quantization of their energy. Therefore, the quantum size effect leads to the electronic properties of the nanosystems which differ from those corresponding to the bulk. In recent years, the interplay between superconductivity and quantum confinement has attracted growing interest due to the unique phenomena which appear if the electron motion is limited to the size less than the coherence length. One of them is the size-dependent enhancement of the energy gap induced by the quantum size effect which has been theoretically investigated by Shanenko et al. in Refs. [7, 8]. Recent experimental results for superconducting nanofilms $[4,5,9-12]$ have shown that the critical temperature and the critical magnetic field oscillate as a function of the nanofilm thickness. Motivated by these experiments, we have studied the critical temperature as a function of the nanofilm thickness. It has been found that for selected nanofilm thicknesses the critical temperature reaches the value that is several times higher as compared to the one measured in the bulk. The enhancement of the critical temperature has been explained on the basis of the quasi-particle energy quantization induced by the electron confinement.

\section{Bogoliubov-de Gennes equations} form

The Bogoliubov-de Gennes (BdG) equations have the

*e-mail: pawel.wojcik@fis.agh.edu.pl

$$
\begin{aligned}
& \left(\begin{array}{cc}
-\frac{\hbar^{2}}{2 m} \nabla^{2}-\mu & \Delta(\boldsymbol{r}) \\
\Delta(\boldsymbol{r}) & \frac{\hbar^{2}}{2 m} \nabla^{2}+\mu
\end{array}\right)\left(\begin{array}{l}
\mathcal{U}_{i}(\boldsymbol{r}) \\
\mathcal{V}_{i}(\boldsymbol{r})
\end{array}\right)= \\
& E_{i}\left(\begin{array}{c}
\mathcal{U}_{i}(\boldsymbol{r}) \\
\mathcal{V}_{i}(\boldsymbol{r})
\end{array}\right),
\end{aligned}
$$

where $\mathcal{U}_{i}(\boldsymbol{r})$ and $\mathcal{V}_{i}(\boldsymbol{r})$ are the electron-like and hole-like wave functions, $E_{i}$ is the quasi-particle energy, $m$ is the free electron mass, $\mu$ is the chemical potential, and $\Delta(\boldsymbol{r})$ is the position-dependent order parameter, which in the absence of the magnetic field, is a real quantity.

The quasi-particle wave functions for the nanofilm can be expressed as

$$
\left(\begin{array}{l}
\mathcal{U}_{k_{x} k_{y} \nu}(\boldsymbol{r}) \\
\mathcal{V}_{k_{x} k_{y} \nu}(\boldsymbol{r})
\end{array}\right)=\frac{\mathrm{e}^{i k_{x} x}}{\sqrt{L_{x}}} \frac{\mathrm{e}^{i k_{y} y}}{\sqrt{L_{y}}}\left(\begin{array}{l}
u_{\nu}(z) \\
v_{\nu}(z)
\end{array}\right),
$$

where $i=\left\{k_{x}, k_{y}, \nu\right\}-k_{x}, k_{y}$ are the free electron wave vector components in the $x$ and $y$ direction (the periodic boundary conditions have been assumed) and $\nu$ labels the subsequent quantum states in the $z$ direction.

The BdG equations with the quasi-particle wave function (2) reduce to the form

$$
\begin{aligned}
& \left(-\frac{\hbar^{2}}{2 m} \frac{\mathrm{d}^{2}}{\mathrm{~d} z^{2}}-\mu+\frac{\hbar^{2} k_{\|}^{2}}{2 m}\right) u_{\nu}(z)+\Delta(z) v_{\nu}(z)= \\
& E_{\nu} u_{\nu}(z), \\
& \left(\frac{\hbar^{2}}{2 m} \frac{\mathrm{d}^{2}}{\mathrm{~d} z^{2}}+\mu-\frac{\hbar^{2} k_{\|}^{2}}{2 m}\right) v_{\nu}(z)+\Delta(z) u_{\nu}(z)= \\
& E_{\nu} v_{\nu}(z),
\end{aligned}
$$

The order parameter $\Delta(z)$ can be expressed in the following manner:

$$
\Delta(z)=\frac{g}{2 \pi} \int \mathrm{d} k_{\|} k_{\|} \sum_{\nu} u_{\nu}(z) v_{\nu}^{*}(z)\left[1-2 f\left(E_{\nu}\right)\right]
$$

where $g$ is the electron-phonon coupling and $f(E)$ is the Fermi-Dirac distribution. The summation in Eq. (5) is 
carried out only over these states for which the singleelectron energy $\xi_{k_{x} k_{y} \nu}$ satisfies the condition $\left|\xi_{k_{x} k_{y} \nu}\right|<$ $\hbar \omega_{\mathrm{D}}$, where $\omega_{\mathrm{D}}$ is the Debye frequency and $\xi_{k_{x} k_{y} \nu}$ is given by

$$
\begin{aligned}
& \xi_{k_{x} k_{y} \nu}= \\
& \quad \int \mathrm{d} z\left[u_{\nu}^{*}(z)\left(-\frac{\hbar^{2}}{2 m} \frac{\mathrm{d}^{2}}{\mathrm{~d} z^{2}}-\mu+\frac{\hbar^{2} k_{\|}^{2}}{2 m}\right) u_{\nu}(z)\right. \\
& \left.\quad+v_{\nu}^{*}(z)\left(-\frac{\hbar^{2}}{2 m} \frac{\mathrm{d}^{2}}{\mathrm{~d} z^{2}}-\mu+\frac{\hbar^{2} k_{\|}^{2}}{2 m}\right) v_{\nu}(z)\right] .
\end{aligned}
$$

The system of Eqs. (3),(4) and Eq. (5) are solved in a self-consistent manner until the convergence is reached. Since the chemical potential for the nanostructures deviates from the bulk value, for each nanofilm thickness we determine the chemical potential using the formula

$$
\begin{aligned}
n_{e} & =\frac{1}{\pi d} \int \mathrm{d} k_{\|} k_{\|} \sum_{\nu} \int \mathrm{d} z \\
& \times\left[\left|u_{\nu}(z)\right|^{2} f\left(E_{\nu}\right)+\left|v_{\nu}(z)\right|^{2}\left(1-f\left(E_{\nu}\right)\right)\right],
\end{aligned}
$$

where $d$ is the thickness of the film in the $z$ direction. The calculations have been carried out for the Al nanofilm with the following values of the parameters: $g N_{\text {bulk }}(0)=0.18$ where $N_{\text {bulk }}(0)=m k_{\mathrm{F}} /\left(2 \pi^{2} \hbar^{2}\right)$ is the bulk density of the single-electron states at the Fermi level, $\hbar \omega_{\mathrm{D}}=32.31 \mathrm{meV}$ and the bulk energy gap $\Delta_{\text {bulk }}=0.25 \mathrm{meV}$.

\section{Results and discussion}

In Fig. 1 we present the critical temperature $T_{\mathrm{C}}$ as a function of the nanofilm thickness $d$. The dependence $T_{\mathrm{C}}(d)$ shows that the critical temperature oscillates as a function of the nanofilm thickness and for some particular values of $d$ abruptly increases reaching the value about six times higher as compared to the critical temperature measured in the bulk $T_{\mathrm{C}}^{\mathrm{bulk}}=1.64 \mathrm{meV}$. The critical temperature for each nanofilm thickness has been determined based on the calculations of the superconducting energy gap $\Delta$ as a function of the temperature $T$.

Figure 2 displays the dependence $\Delta(T)$ for four chosen nanofilm thicknesses: $d=1.08 \mathrm{~nm}$ which corresponds to the first maximum of $T_{\mathrm{C}}, d=1.7 \mathrm{~nm}$ which corresponds to the drop of the critical temperature below its bulk value, $d=1.76 \mathrm{~nm}$ and $d=2.44 \mathrm{~nm}$ which correspond to the second and third maximum of $T_{\mathrm{C}}$. The chosen nanofilm thicknesses are marked in Fig. 1a by arrows. The enhancement of the critical temperature for some nanofilm thicknesses can be understood on the basis of the analysis of the interplay between the superconductivity and the quantum size effect. In the ultrathin nanofilm, the confinement of electrons in the direction perpendicular to the surface leads to the quantization of their energy. It means that the Fermi sphere splits into a series of parabolic subbands. Futhermore, it is well known that the Cooper-pair condensation is determined by the electron-phonon interaction and concerns only electrons from the energy window $\left[\mu-\hbar \omega_{\mathrm{D}}, \mu+\hbar \omega_{\mathrm{D}}\right]$

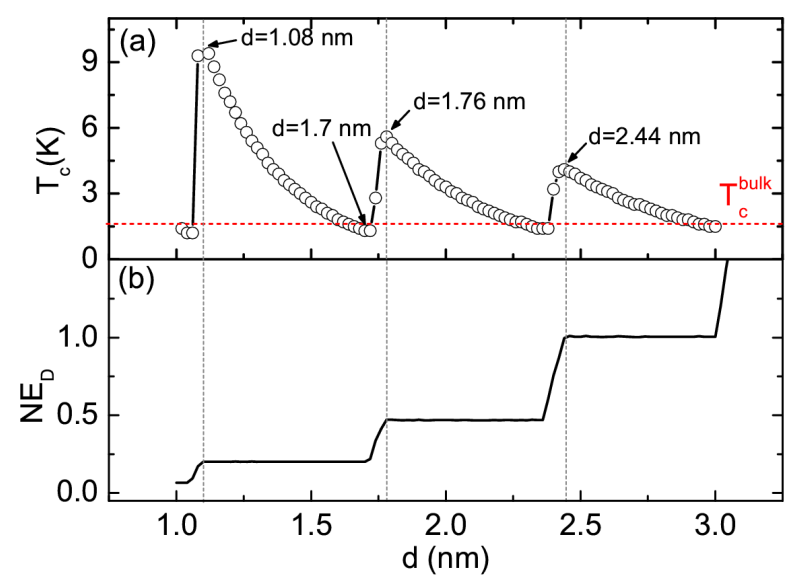

Fig. 1. (a) Critical temperature $T_{\mathrm{C}}$ and (b) electron density of states $N$ in the energy window $E_{\mathrm{D}}=$ $\left[\mu-\hbar \omega_{\mathrm{D}}, \mu+\hbar \omega_{\mathrm{D}}\right]$ as a function of the nanofilm thickness $d$. In part (a) the critical temperature for the bulks is marked by the dashed red line.

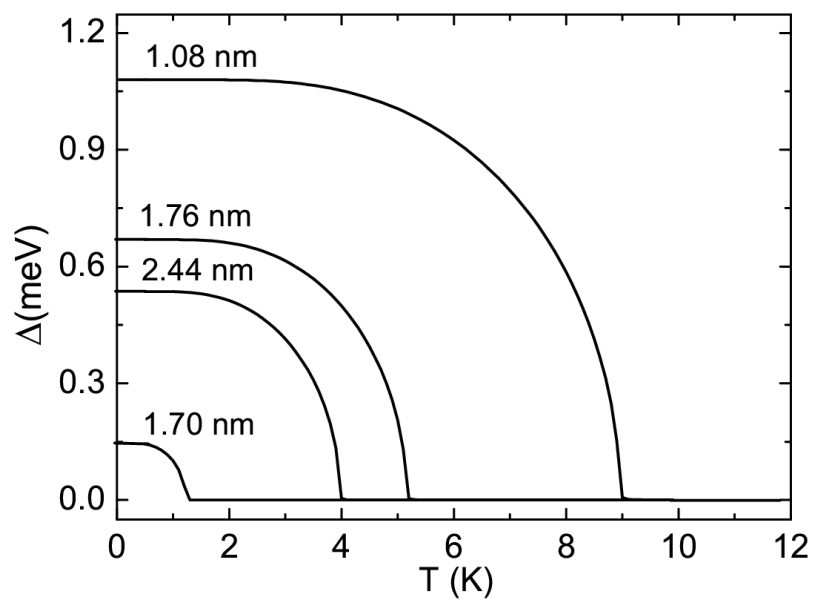

Fig. 2. The superconducting energy gap $\Delta$ as a function of temperature $T$ for nanofilm thicknesses marked by arrows in Fig. 1a.

around the Fermi level, where $\omega_{\mathrm{D}}$ is the Debye frequency. Therefore the superconducting gap strongly depends on the number of states in the mentioned energy range. In the ultrathin nanofilm, the increase of the thickness $d$ leads to decrease of the energy of each parabolic subbands. If the subsequent subbands pass through the energy window $\left[\mu-\hbar \omega_{\mathrm{D}}, \mu+\hbar \omega_{\mathrm{D}}\right]$, we can observe the step-like enhancement of the density of states participating in the condensation of the Cooper-pairs (see Fig. 1b). The described mechanism causes the increase of the zero-temperature energy gap and consequently the enhancement of the critical temperature for particular nanofilm thicknesses. The enhancement of the zero-temperature energy gap is clearly visible in Fig. 2.

In order to determine the number of subbands which participate in the Cooper-pair condensation, in Fig. 3 we 


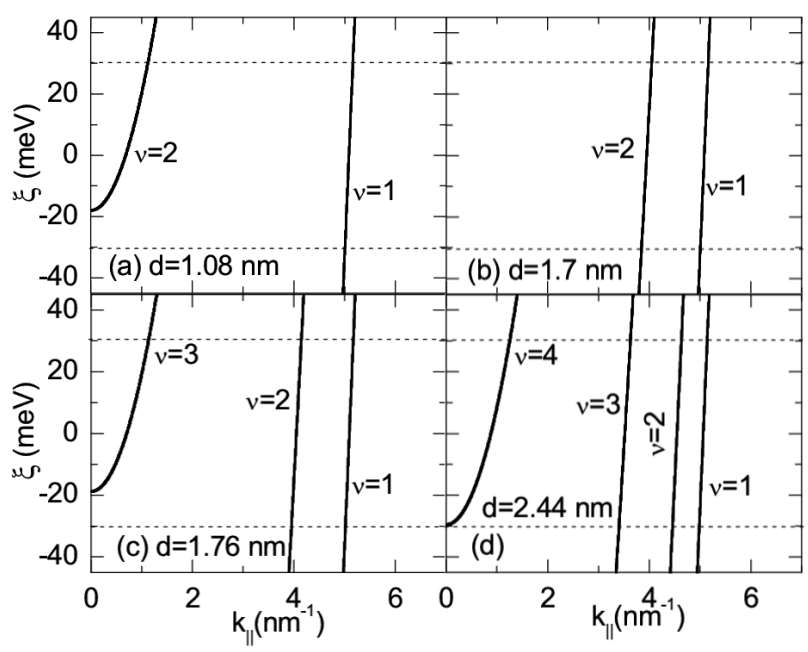

Fig. 3. Quasi-particle kinetic energy $\xi$ as a function of the wave vector for nanofilm thicknesses $d$ marked by arrows in Fig. 1a.

present the quasi-particle kinetic energy $\xi$ as a function of the wave vector $k_{||}$for considered nanofilm thicknesses (see Fig. 1a). The energy window $\left[\mu-\hbar \omega_{\mathrm{D}}, \mu+\hbar \omega_{\mathrm{D}}\right]$ around the Fermi level has been marked by the dashed horizontal lines. We can see that for the nanofilm thickness $d=1.08 \mathrm{~nm}$ (Fig. 3a) the enhancement of the critical temperature corresponds to the Cooper pairing of electrons from the quantum subband $\nu=2$ which kinetic energy minimum is located in the energy window $\left[\mu-\hbar \omega_{\mathrm{D}}, \mu+\hbar \omega_{\mathrm{D}}\right]$. By analogy, Fig. 3c and d allows us to conclude that the second and third maximum correspond to the condensation of electrons from the subband $\nu=3$ and $\nu=4$, respectively. In contrary, in Fig. 3b we can observe that the drop of critical temperature below its bulk value results from the fact that the minimum of the subband $\nu=2$ leaves the energy window $\left[\mu-\hbar \omega_{\mathrm{D}}, \mu+\hbar \omega_{\mathrm{D}}\right]$.

\section{Conclusions}

The critical temperature as a function of the nanofilm thickness has been studied on the basis of the selfconsistent numerical solution of BdG equations. Calculations for $\mathrm{Al}$ nanofilms have shown that for some nanofilms thicknesses the critical temperature increases up to the value six times higher as compared to the critical temperature in the bulk. The enhancement of the critical temperature has been explained based on the quantization of the electron energy in the direction perpendicular to the plane. Our results are in agreement with the oscillations of the critical temperature experimentally observed for the superconducting nanofilms.

\section{Acknowledgments}

This work was financed from the budget for Polish Science in the years 2013-2014. Project number: IP2012 048572 .

\section{References}

[1] L. Janković, D. Gournis, P.N. Trikalitis, I. Arfaoui, T. Cren, P. Rudolf, M.H. Sage, T.T.M. Palstra, B. Kooi, J. De Hosson, A. Karakassides, K. Dimos, A. Moukarika, T. Bakas, Nano Lett. 6, 1131 (2006).

[2] F. Altomare, A.M. Chang, M.R. Melloch, Y. Hong, C.W. Tu, Phys. Rev. Lett. 97, 017001 (2006).

[3] M. Zgirski, K.-P. Riikonen, V. Touboltsev, K. Arutyunov, Nano Lett. 5, 1029 (2005).

[4] Y. Guo, Y.-F. Zhang, X.-Y. Bao, T.-Z. Han, Z. Tang, L.-X. Zhang, W.-G. Zhu, E. G. Wang, Q. Niu, Z.Q. Qiu, J.-F. Jia, Z.-X Zhao, Q.-K. Xue, Science 306, 1915 (2004).

[5] M.M. Özer, J.R. Thompson, H.H. Weitering, Nature Phys. 2, 173 (2006).

[6] M.-L. Tian, J.-G. Wang, J.S. Kurtz, Y. Liu, M.H.W. Chan, T.S. Mayer, T.E. Mallouk, Phys. Rev. $B$ 71, 104521 (2005).

[7] A.A. Shanenko, M.D. Croitoru, M. Zgirski, F.M. Peeters, K. Arutyunov, Phys. Rev. B 74, 052502 (2006).

[8] A.A. Shanenko, M.D Croitoru, Phys. Rev. B 73, 012510 (2006).

[9] M.M. Özer, J.R Thompson, H.H. Weitering, Phys. Rev. B 74, 235427 (2006).

[10] M.M. Özer, Y. Jia, Z. Zhang, J.R. Thompson, H.H. Weitering, Science 316, 1594 (2007).

[11] T. Zhang, P. Cheng, W.J. Li, Y.J. Sun, G. Wang, X.G. Zhu, K. He, L. Wang, X. Ma, X. Chen, Y. Wang, Y. Liu, H. Q. Lin, J.F. Jia, Q.K Xue, Nature Phys. 6, $104(2010)$.

[12] S. Qin, J. Kim, Q. Niu, C.K. Shih, Science 324, 1314 (2009). 